Proyecciones

Vol. 22, $\mathrm{N}^{o}$ 1, pp. 81-89, May 2003.

Universidad Católica del Norte

Antofagasta - Chile

DOI: $10.4067 /$ S0716-09172003000100005

\title{
ON CERTAIN PROPERTIES OF SOME GENERALIZED SPECIAL FUNCTIONS
}

\author{
M. A. PATHAN and SUBUHI KHAN \\ Aligarh Muslim University, India
}

\begin{abstract}
In this paper, we derive a result concerning eigenvector for the product of two operators defined on a Lie algebra of endomorphisms of a vector space. The results given by Radulescu, Mandal and authors follow as special cases of this result. Further using these results, we deduce certain properties of generalized Hermite polynomials and Hermite Tricomi functions.
\end{abstract}

2000 Mathematics Subject Classification : 33C45, 33C50, 33C80.

Keywords : Generalized Special Functions, Lie Algebra. 


\section{Introduction}

The interplay between differential equations, generalized special functions and Lie theory is particularly useful in applications. The theory of generalized special functions has witnessed a rather significant evolution during the last years (see Dattoli et al. $[1,2,3,4]$ ). In applicative fields, we note that for some physical problems the use of new classes of special functions provided solutions hardly achievable with conventional analytical and numerical means.

Radulescu [8] has discussed some properties of Hermite and Laguerre polynomials [9] using some operators defined on a Lie algebra. Further Mandal [6] obtained some properties of simple Bessel polynomials considered by Krall and Frink [5]. Recently Pathan and Khan [7] discussed some properties of two variable Laguerre polynomials (TVLP) studied by Dattoli and Torre $[3,4]$.

In this paper, we establish a result concerning eigenvector for the product of two operators defined on a Lie algebra of endomorphisms of the vector space $V$. The results given by Radulescu [8], Mandal [6] and Pathan and Khan [7] follow as special cases of this result.

Further, we extend the approach of Radulescu and Mandal $[8,6]$ to deduce some properties of two variable Hermite-Kampé de Fériét polynomials (TVHKdFP) and Hermite-Tricomi functions (HTF). The analytic methodology developed in this paper can easily be adopted to the study of some other special functions of mathematical physics.

The TVHKdFP $H_{n}(x, y)[2]$ are specified by the series

$$
H_{n}(x, y)=\sum_{r=0}^{[n / 2]} \frac{n ! y^{r} x^{n-2 r}}{r !(n-2 r) !},
$$

and the generating function for $H_{n}(x, y)$ is given by

$$
\sum_{n=0}^{\infty} H_{n}(x, y) \frac{t^{n}}{n !}=\exp \left(x t+y t^{2}\right) .
$$

The polynomials $h_{n}(x, y ; \xi)[2]$ generated by

$$
\sum_{n=0}^{\infty} h_{n}(x, y ; \xi) \frac{t^{n}}{n !}=\exp \left(2 x t-t^{2}+2 y \xi t-\xi^{2} t^{2}\right),
$$

can be written in terms of $H_{n}(x, y)$ as follows :

$$
h_{n}(x, y ; \xi)=H_{n}\left(2(x+y \xi),-\left(1+\xi^{2}\right)\right) .
$$


The use of $h_{n}(x, y ; \xi)$ is particularly useful for obtaining a closed expression for the infinite sum

$$
\sum_{n=0}^{\infty} \frac{t^{j n} H_{j n+m}(x)}{(p n) !} .
$$

Further we note that the $\mathrm{HTF}_{H} C_{n}(x, y)$ are defined by the series ([4]; p.24 $(36 \mathrm{~b}))$

$$
{ }_{H} C_{n}(x, y)=\sum_{s=0}^{\infty} \frac{(-1)^{s} H_{s}(x, y)}{s !(n+s) !}
$$

and the generating function for ${ }_{H} C_{n}(x, y)$ is given as ([4]; p.25 (47))

$$
\sum_{n=-\infty}^{\infty}{ }_{H} C_{n}(x, y) t^{n}=\exp \left(t-\frac{x}{t}+\frac{y}{t^{2}}\right) .
$$

For $y=0$, the $\operatorname{HTF}{ }_{H} C_{n}(x, y)$ reduces to the Tricomi functions $C_{n}(x)$, which are linked to the ordinary Bessel functions by the relation [1]

$$
C_{n}(x)=x^{-n / 2} J_{n}(2 \sqrt{x}) .
$$

\section{Main Theorem}

Let End $V$ be the Lie algebra of endomorphisms of the vector space $V$, endowed with the Lie bracket $[\cdot, \cdot]$ defined by $[A, B]=A B-B A$, for every $A, B \in$ End $V$. We denote by $I$ the identity operator of $V$.

The main theorem of the paper is as follows.

Theorem -1: Let $A, B \in$ End $V$ be such that $[A, B] y_{n}=(a(2 n+1)+$ b) $y_{n}$, where the sequence $\left(y_{n}\right)_{n} \subset V$ is defined as follows: $A y_{1}=y_{0}$ and

$B y_{n}=\left(\frac{\left(a\left(n^{2}+2 n\right)+b n+1\right)}{(a n+b n+1)}\right) y_{n+1}$, for every $n \geq 0$. Then $A y_{n+1}=(a n+b n+$ 1) $y_{n}$, and $y_{n}$ is an eigenvector of eigenvalue $\left(a\left(n^{2}-1\right)+b(n-1)+1\right)$ for $B A$, for every $n \geq 1$.

Proof. First we show that

$$
A y_{n+1}=(a n+b n+1) y_{n}, \text { for every } n \geq 1 \text {. }
$$

We have

$$
\begin{gathered}
{[A, B] y_{1}=(3 a+b) y_{1},} \\
A\left(B y_{1}\right)-B\left(A y_{1}\right)=(3 a+b) y_{1} .
\end{gathered}
$$


Also, $A y_{1}=y_{0}, \quad B y_{0}=y_{1}$ and $B y_{1}=\frac{(3 a+b+1)}{(a+b+1)} y_{2}$, and therefore $A y_{2}=$ $(a+b+1) y_{1}$.

Next, suppose that $A y_{n+1}=(a n+b n+1) y_{n}$. Now since we may write

$$
\begin{gathered}
{[A, B] y_{n+1}=(a(2 n+3)+b) y_{n+1},} \\
A\left(B y_{n+1}\right)-B\left(A y_{n+1}\right)=(a(2 n+3)+b) y_{n+1}, \\
\frac{\left(a\left((n+1)^{2}+2(n+1)\right)+b(n+1)+1\right)}{(a(n+1)+b(n+1)+1)} A y_{n+2}-(a n+b n+1) B y_{n} \\
=(a(2 n+3)+b) y_{n+1}, \\
\frac{\left(a\left(n^{2}+4 n+3\right)+b(n+1)+1\right)}{(a(n+1)+b(n+1)+1)} A y_{n+2}-\left(a\left(n^{2}+2 n\right)+b n+1\right) y_{n+1} \\
=(a(2 n+3)+b) y_{n+1}, \\
\frac{\left(a\left(n^{2}+4 n+3\right)+b(n+1)+1\right)}{(a(n+1)+b(n+1)+1)} A y_{n+2} \\
A y_{n+2}=(a(n+1)+b(n+1)+1) y_{n+1} .
\end{gathered}
$$

Hence by mathematical induction $A y_{n+1}=(a n+b n+1) y_{n}$, for every $n \geq 1$. It follows that

$$
(B A) y_{n}=(a(n-1)+b(n-1)+1) B y_{n-1},
$$

i.e.

$$
(B A) y_{n}=\left(a\left(n^{2}-1\right)+b(n-1)+1\right) y_{n} .
$$

Hence $y_{n}$ is an eigenvector of eigenvalue $\left(a\left(n^{2}-1\right)+b(n-1)+1\right)$ for $B A$, for every $n \geq 1$.

\section{Deductions and Applications of Theorem 1.}

We note that, for $a=0, b=1$, Theorem-1 yields the main result of Radulescu ([8] ; p.67 (Theorem-1)). Again for $a=0, b=0$, it yields the main result of Mandal ([6] ; p. 273 (Theorem-1)). And finally for $a=1$ and $b=0$, it gives the main result of Pathan and Khan [7].

First, we consider the case, when $a=0$ and $b=1$, that is, we recall the following main theorem of Redulescu [8]:

Theorem-2: Let $C, D \in$ End $V$ be such that $[C, D]=I$. We define the sequence $\left(Z_{n}\right)_{n} \subset V$ as follows: $C Z_{0}=0$ and $Z_{n}=D Z_{n-1}$, for every $n \geq 1$. Then $C Z_{n}=n Z_{n-1}$ and $Z_{n}$ is an eigenvector of eigenvalue $n$ for $D C$, for every $n \geq 1$. 
Let $V=C^{\infty}(\mathbb{R} \times \mathbb{R})$. We define the operators $C, D \in$ End $V$ by

$$
\begin{aligned}
& C f(x, y)=f^{\prime}(x, y), \\
& D f(x, y)=2 y f^{\prime}(x, y)+x f(x, y),
\end{aligned}
$$

for every $(x, y) \in \mathbb{R} \times \mathbb{R}$, and where $f^{\prime}$ denotes $\frac{\partial f}{\partial x}$. It can be easily seen that these operators satisfy the commutation relation $[C, D]=I$.

Next, we prove that

$$
D^{n} f(x, y)=(-1)^{n}(-y)^{n / 2}\left[e^{t^{2}}\left(f(x, y) e^{-t^{2}}\right)^{(n)} \mid t=\frac{x}{2 \sqrt{-y}}\right] .
$$

From the definition of $D$, the above equality holds for $n=1$. Inductively, taking into account $D^{n+1} f(x, y)=D\left(D^{n} f(x, y)\right)$, it follows that

$$
D^{n+1} f(x, y)=(-1)^{n+1}(-y)^{\frac{(n+1)}{2}}\left[e^{t^{2}}\left(f(x, y) e^{-t^{2}}\right)^{(n+1)} \mid t=\frac{x}{2 \sqrt{-y}}\right],
$$

which ends our proof.

The TVHKdFP, $H_{n}(x, y)$ satisfies the following differential equation

$$
2 y Z^{\prime \prime}(x, y)+x Z^{\prime}(x, y)-n Z(x, y)=0,
$$

where $n$ is a positive integer.

Equation (3.3) may be written as

$$
2 y Z^{\prime \prime}(x, y)+x Z^{\prime}(x, y)=n Z(x, y),
$$

which by using (3.1) reduces to

$$
D Z^{\prime}(x, y)=n Z(x, y)
$$

Therefore

$$
D C Z(x, y)=n Z(x, y) .
$$

By Theorem-2, it follows that $Z_{n}(x, y)$ is a solution of the differential equation (3.3). Setting $Z_{0}(x, y)=1$, we obtain $Z_{n}(x, y)=D^{n}(1)$. Therefore defining $H_{n}(x, y)=Z_{n}(x, y)$, we deduce the Rodrigues-type formula

$$
H_{n}(x, y)=(-1)^{n}(-y)^{n / 2}\left[e^{t^{2}}\left(\frac{d}{d t}\right)^{n} e^{-t^{2}} \mid t=\frac{x}{2 \sqrt{-y}}\right] .
$$

Proceeding in this manner we are able to deduce other properties of 
TVHKdFP $H_{n}(x, y)$.

Since $H_{n}(x, y)=Z_{n}(x, y)=D^{n}(1)$, we can use the equality $C Z_{n}=$ $n Z_{n-1}$. Now from the definition of the operator $C$, it follows that

$$
Z_{n}^{\prime}(x, y)=n Z_{n-1}(x, y)
$$

i.e.

$$
\frac{\partial}{\partial x} H_{n}(x, y)=n H_{n-1}(x, y)
$$

Again, by the definition of the operator $D$, we have

$$
D Z_{n}(x, y)-2 y Z_{n}^{\prime}(x, y)-x Z_{n}(x, y)=0,
$$

which by using (3.4) can be written as

$$
Z_{n+1}(x, y)-2 n y Z_{n-1}(x, y)-x Z_{n}(x, y)=0,
$$

or equivalently

$$
H_{n+1}(x, y)+x H_{n}(x, y)-2 n y H_{n-1}(x, y)=0 .
$$

Further, we may derive corresponding properties for $h_{n}(x, y ; \xi)$ using (1.4).

Next, we consider the case when $a=0$ and $b=0$, that is, we consider the following theorem of Mandal [6] :

Theorem-3: Let $E, F \in$ End $V$ be such that $[E, F]=0$. We define the sequence $\left(W_{n}\right)_{n} \subset V$ as follows: $E W_{1}=W_{0}$ and $W_{n+1}=F W_{n}$ for every $n \geq 0$. Then $E W_{n+1}=W_{n}$ for every $n \geq 1$ and $W_{n}$ is an eigenvector of eigenvalue 1 for $F E$ for every $n \geq 1$.

Let $V=C^{\infty}(\mathbb{R} \times \mathbb{R} \times \mathbb{R})$. We define the operators $E, F \in$ End $V$ by

$$
\begin{aligned}
& E u(x, y, t)=\frac{x}{t} u_{x}+\frac{2 y}{t} u_{y}+u_{t}, \\
& F u(x, y, t)=-t u_{x},
\end{aligned}
$$

for every $(x, y, t) \in \mathbb{R} \times \mathbb{R} \times \mathbb{R}$, where $u_{x}, u_{y}$ and $u_{t}$ denote $\frac{\partial u}{\partial x}, \frac{\partial u}{\partial y}$ and $\frac{\partial u}{\partial t}$ respectively. It can be easily seen that these operators satisfy the commutation relation $[E, F]=0$.

Now if $u(x, y, t)$ assumes the form $W_{n}(x, y, t)=V_{n}(x, y) t^{n} \in C^{\infty}(\mathbb{R} \times$ $\mathbb{R} \times \mathbb{R}$ ), then $F W_{n}=W_{n+1} \Longrightarrow E W_{n+1}=W_{n}$ by virtue of Theorem-3. Now the relation $F W_{n}=W_{n+1}$ yields

$$
\left(-t \frac{\partial}{\partial x}\right)\left(V_{n}(x, y) t^{n}\right)=V_{n+1}(x, y) t^{n+1}
$$


i.e.

$$
\frac{\partial}{\partial x} V_{n}(x, y)=-V_{n+1}(x, y)
$$

Again the relation $E W_{n+1}=W_{n}$ yields

$$
\left(\frac{x}{t} \frac{\partial}{\partial x}+\frac{2 y}{t} \frac{\partial}{\partial y}+\frac{\partial}{\partial t}\right)\left(V_{n}(x, y) t^{n}\right)=V_{n-1}(x, y) t^{n-1},
$$

i.e.

$$
\left(x \frac{\partial}{\partial x}+2 y \frac{\partial}{\partial y}+n\right) V_{n}(x, y)=V_{n-1}(x, y),
$$

which by using (3.7) can be written as

$$
x V_{n+1}(x, y)-\left(2 y \frac{\partial}{\partial y}+n\right) V_{n}(x, y)+V_{n-1}(x, y)=0,
$$

Again by virtue of Theorem-3, we can write

$$
F E W_{n}(x, y, t)=W_{n}(x, y, t)
$$

i.e.

$$
\left(-t \frac{\partial}{\partial x}\right)\left(\frac{x}{t} \frac{\partial}{\partial x}+\frac{2 y}{t} \frac{\partial}{\partial y}+\frac{\partial}{\partial t}\right)\left(V_{n}(x, y) t^{n}\right)=V_{n}(x, y) t^{n}
$$

i.e.

$$
\left(x \frac{\partial^{2}}{\partial x^{2}}+2 y \frac{\partial^{2}}{\partial x \partial y}+(n+1) \frac{\partial}{\partial x}+1\right) V_{n}(x, y)=0 .
$$

Now it is evident from the differential equation (3.10) that $\mathrm{HTF}{ }_{H} C_{n}(x, y)$ (1.5)-(1.6) is a solution of the above differential equation. It is interesting to note that (3.7), (3.8) and (3.9) are differential recurrence relations satisfied by ${ }_{H} C_{n}(x, y)$.

Acknowledgement: The second author is thankful to The Abdus Salam International Center for Theoretical Physics (ICTP), Trieste, for hospitality and financial support for the period of her stay as visiting fellow.

\section{References}

[1] G. Dattoli and A. Torre; Theory and Applications of Generalized Bessel Functions, ARACNE, Rome (1996). 
[2] G. Dattoli, A. Torre and M. Carpanese; Operational rules and arbitrary order Hermite generating functions, J. Math. Anal. Appl., 227, pp. 98-111, (1998).

[3] G. Dattoli and A. Torre; Operational methods and two variable Laguerre polynomials, Acc. Sc. Torino-Atti Sc. Fis., 132, pp. 1-7, (1998).

[4] G. Dattoli and A. Torre; Exponential operators, quasi-monomials and generalized polynomials, Radiation Physics and Chemistry, 57, pp. 21-26, (2000).

[5] H. L. Krall and O. Frink; A new class of orthogonal polynomials: the Bessel polynomials, Trans. Amer. Math. Soc., 65, pp. 100-115, (1949).

[6] A. K. Mandal; Some operators on a Lie algebra and simple Bessel polynomials, Soochow J. Math., 25, No. 3, pp. 273-276, (1999).

[7] M. A. Pathan and Subuhi Khan; Some properties of two variable Laguerre polynomials via Lie algebra, To appear in Integral Transforms and Special Functions, (2002-2003).

[8] V. D. Radulescu; A study of some special functions with Lie theory, Stud. Cerc. Mat., 43, No. 1-2, pp. 67-71, (1991).

[9] H. M. Srivastava and H. L. Manocha; A Treatise on Generating Functions, Ellis Horwood Limited, Chichester, New York, (1984).

Received : March 2002.

\section{Mahmood Ahmad Pathan}

Department of Mathematics

Aligarh Muslim University

Aligarh 202002

India

e-mail : mapathan@postmark.net

and 


\section{Subuhi Khan}

Department of Mathematics

Aligarh Muslim University

Aligarh 202002

India

e-mail : subuhi2000@postmark.net 